\title{
SEXTING PRACTICADO POR ADOLESCENTES: SU MORFOLOGÍA EN FACEBOOK
}

\author{
Mercado Contreras Cinthia Tomasa, \\ Universidad Autónoma de Aguascalientes \\ Cervantes Herrera Ana del Refugio \\ Universidad Autónoma de Ciudad Juárez \\ https://doi.org/10.17060/ijodaep.2017.n1.v2.932
}

Fecha de Recepción: 23 Marzo 2017

Fecha de Admisión: 1 Abril 2017

\section{RESUMEN}

En el presente trabajo se presentan los resultados de un análisis exploratorio y descriptivo, a través de un método observacional de diversos episodios de sexting tomando como base la teoría verbal de Skinner.

Para llevarlo a cabo se desarrolló un sistema de categorización ad hoc a los mandos presentados en la conducta verbal del sexting en Facebook, pudiendo con ello observar, codificar y analizar el fenómeno, mismo que ha tomado auge en los últimos cinco años, sobre todo en los adolescentes.

Tomando como base la teoría de Skinner se consideraron las diferentes opciones de mandos que los participantes usan por vía inbox y la similitud entre algunos de ellos. Por medio de 9 conversaciones revisadas por dos observadores se obtuvo un nivel sustancial y de fiabilidad en la herramienta, esto de acuerdo al índice Kappa de Cohen $(k=.86)$. El objetivo del trabajo fue describir, a partir de dichas categorías, la conducta verbal en episodios de sexting, considerando los mandos emitidos, la frecuencia de emisión, sus probabilidades de transición y los patrones desplegados por los participantes.

Palabras Clave: sexting, conducta verbal, observación, patrones, conversación.

\section{ABSTRACT}

\section{Construction tool observacional of verbal conduct in sexting's conversations}

En el presente trabajo se presentan los resultados de un análisis exploratorio y descriptivo, a través de un método observacional de diversos episodios de sexting tomando como base la teoría verbal de Skinner.

Para llevarlo a cabo se desarrolló un sistema de categorización ad hoc a los mandos presentados en la conducta verbal del sexting en Facebook, pudiendo con ello observar, codificar y analizar el fenómeno, mismo que ha tomado auge en los últimos cinco años, sobre todo en los adolescentes. 


\section{SEXTING PRACTICADO POR ADOLESCENTES: SU MORFOLOGÍA EN FACEBOOK}

Tomando como base la teoría de Skinner se consideraron las diferentes opciones de mandos que los participantes usan por vía inbox y la similitud entre algunos de ellos. Por medio de 9 conversaciones revisadas por dos observadores se obtuvo un nivel sustancial y de fiabilidad en la herramienta, esto de acuerdo al índice Kappa de Cohen $(k=.86)$. El objetivo del trabajo fue describir, a partir de dichas categorías, la conducta verbal en episodios de sexting, considerando los mandos emitidos, la frecuencia de emisión, sus probabilidades de transición y los patrones desplegados por los participantes.

Palabras Clave: sexting, conducta verbal, observación, patrones, conversación.

\section{ANTECEDENTES}

El sexting es un fenómeno que ha sido revisado y analizado por investigadores, profesionales de la salud, profesionales en legalidad y maestros (Farber, Shafron, Hamadani, Wald y Nitzburg, 2012; Yang, 2015). Dicho fenómeno no cuenta, en la actualidad, con una definición consensuada, sin embargo las características más mencionadas por diferentes autores es que se trata del intercambio de mensajes en alguna conversación privada (inbox) por medio de algún medio electrónico, con contenido sexual explícito o implícito, ya sea con texto y/o imagen creada por el autor, en donde se muestra desnudo o semidesnudo (Lounsbury, Mitchell y Finkelhor, 2011; Marrufo, 2012; Menjívar, 2010; Wolak y Finkelhor, 2011).

La observación de dicho fenómeno ha ido en aumento a partir de las consecuencias negativas observadas en sus participantes (Farber et al., 2012; Marrufo, 2012), además de que los teléfonos móviles se están convirtiendo en una extensión natural del cuerpo humano por la relación tan estrecha que tiene el propietario con dicho aparato (Zemmels y Khey, 2014).

El aumento de la revisión del fenómeno del sexting se ha visto influido por la cantidad de consecuencias legales que su práctica implica, especialmente considerando lo que Yang (2015) indica que los autores de "Adolescent Sexual Behavior in the Digital Age" llaman el pánico moral y jurídico, refiriéndose a las consecuencias que a principios del 2010 fueron consideradas graves, éstas dadas a partir del registro que existe en la Internet de la actividad que se realiza, sobre todo por la inclusión de las imágenes en los mensajes enviados, ya que éstas pueden persistir en la Internet y, con ello, existe la posibilidad de ser visibles para personas no autorizadas por el autor (Arab y Díaz, 2015; Wolak y Finkelhor, 2011; Yang, 2015). A pesar de ello, la legislación no ha podido determinar específicamente una pena o siquiera una definición del sexting que permita su inclusión tipificada (Drouin, Vogel, Surbey y Stills, 2013).

Otra de las dificultades que se ha presentado, desde que el fenómeno es de interés para la investigación, además de la gran variedad de definiciones de sexting, es la difícil comparación de datos estudiados debido a las diferentes metodologías que usan los autores (Anderson, Fagan, Woodnutt y Chamorrro-Premuzic, 2012; Lounsbury, Mitchell y Finkelhor, 2011; Zemmels y Khey, 2014).

De acuerdo con Delevi y Weisskirch (2013) los estudios futuros sobre el tema de sexting deberían ser tratados desde una perspectiva más objetiva de la conducta, tomando en cuenta una revisión más directa de las conversaciones y sus historiales, así como informes de la participación del destinatario.

Bajo la visión de una mejor comprensión del problema, tanto profesionales de la salud, como legisladores tendrían la posibilidad de ofrecer una mejor educación y protección a los usuarios de la Internet para el uso de las nuevas tecnologías (Arab y Díaz, 2015), sobre todo a los niños y adolescentes, quienes han sido considerados los más vulnerables de todas las consecuencias negativas observadas (Farber, et al., 2012).

Peña-Correal y Robayo-Castro (2007) proponen la Conducta Verbal para el estudio de fenómenos relacionados con el lenguaje, y siendo que el sexting se trata de una expresión escrita y que se 
observa solamente en conversaciones privadas, su propuesta es relevante para el su estudio, sobre todo desde una perspectiva de la Psicología

Desde la perspectiva conductual, el lenguaje es moldeado a partir de la comunidad de hablantes en la que se encuentran inmersos los participantes, y a partir de las reglas dadas por ellos se establece una manera objetiva y concreta de analizarlo (Skinner, 1979). Una característica importante del lenguaje, que se toma en cuenta desde esta perspectiva, es la interacción que se presenta entre el hablante y el oyente, de la cual es preciso hacer una descripción de ambas partes para poder realizar una explicación completa de la conducta verbal (Skinner, 1981).

En dicha interrelación es posible diferenciar ciertas variantes, entre ellas la competencia, el altruismo, la confianza y el compartir (Santoyo, Cortés, Torres y Espinosa, 1985), las cuales tienen sus características basadas en la oportunidad que tiene el hablante y el oyente de realizar una tarea, el otorgamiento de reforzadores entre ellos y la distribución de participaciones dentro de la interrelación.

De acuerdo con Skinner (1981), la relación entre el hablante y el escucha puede darse de cuatro maneras diferentes: i) ecoica, cuando la conducta está bajo estímulos verbales; ii) textual, en donde la conducta es presentada por medio de dibujos, pictogramas formales, jeroglíficos, caracteres, símbolos o letras de un alfabeto fonético; iii) intraverbal, es decir, los estímulos no tienen una correspondencia formal en algo vocal o escrito y; iv) tacto, en la cual el estímulo es un objeto o evento particular.

En el presente trabajo se tomará en cuenta la conducta de manera textual, siendo analizada como una conducta ecoica pero con estímulos visuales en lugar de auditivos. En dicha conducta el hablante es el escritor y el escucha es el lector, sin embargo, ambos tienen una participación dual durante todo el episodio verbal completo.

Al considerase el lenguaje como prioritario en nuestra especie, un tratamiento skinneriano del fenómeno, se considera una base desde una perspectiva psicológica para poder explicar el comportamiento (Ardila, 2007), además de ser éste un camino para tener un mejor conocimiento del sexting y la relación funcional observable en su práctica. A partir de dicho conocimiento se posibilitaría una predicción de las respuestas, auxiliar en una propuesta de cambio y difusión de un buen uso de las Tecnologías de Información y Comunicación (TICs), y se estaría en posibilidades de ofrecer capacitación, sobre todo a los más jóvenes, para un mejor control de sus respuestas en las redes sociales.

La metodología observacional es pertinente para este estudio ya que el fin del mismo cumple con las cuatro características fundamentales del perfil mencionadas por Anguera y HernándezMendo (2014), es decir, se trata de un estudio: 1. De comportamiento espontáneo o habitual; 2 dentro de un contexto natural; 3 . Con el uso de instrumentos no estándar; y 4. Con conducta totalmente perceptible.

Es por ello que se presenta una investigación en donde el objetivo es describir el fenómeno del sexting a partir de un análisis funcional de la conducta verbal en episodios de sexting, considerando los mandos emitidos por los sujetos, la frecuencia de emisión de éstos mandos, las probabilidades de transición entre ellos y finalmente, los patrones desplegados por los participantes.

\section{MÉTODO}

\section{Diseño}

Para cumplimentar el objetivo de esta investigación se realizó un estudio exploratorio y descriptivo, esto a través de un método observacional con un diseño transversal. 


\section{SEXTING PRACTICADO POR ADOLESCENTES: SU MORFOLOGÍA EN FACEBOOK}

\section{Muestra}

Para el presente trabajo se tomaron en cuenta para el análisis 9 conversaciones de sexting de 3 diferentes participantes que enviaron voluntariamente sus conversaciones con interlocutores diferentes.

\section{Material}

Cuestionario de Sexo y Tecnología.- Traducción y adaptación, realizada por Marrufo (2012) del cuestionario llamado "Sex and Tech". Para la presente investigación se eliminaron los ítems correspondientes a las razones por las que se cometería el Sexting y las consecuencias que preocuparían al adolescente que lo cometería, dando un total de 37 ítems sobre "Percepción general del adolescente sobre cómo se da el Sexting", "Acciones del Sexting cometidas"y "Postura ante afirmaciones sobre el Seting".

Catálogo Conductual. La clasificación que se propone se fundamenta en el tipo de mando y reforzadores que Skinner identifica (1981a) haciendo una diferencia en la modalidad de mensaje, esto tomando en cuenta la definición de la práctica del Sexting que considera tanto el texto como la imagen, dejando de lado la modalidad de video por tratarse de un elemento poco considerado en la definición ofrecida por los diferentes autores revisados. También se incluye una diferenciación en la forma del texto 0 imagen, es decir, si se presenta implícita 0 explícitamente, esto de acuerdo a la teoría de la Conducta Verbal que sugiere que dependiendo de la audiencia será el repertorio verbal.

Un par de observadores fueron entrenados para la obtención de datos. El Catálogo Conductual obtuvo una confiabilidad de un $88.88 \%$ de índice de acuerdos, ofreciendo un coeficiente Kappa de $.86(p<.0001)$

\section{Procedimiento}

Al tratarse de un fenómeno expuesto en la Internet se tomó como plataforma la red social Facebook, considerada de mayor popularidad entre jóvenes y adolescentes, y que además permite a los usuarios guardar las conversaciones por tiempo indefinido para su consulta en tiempo diferido (Cuesta y Gaspar, 2013).

Se consideró al sexting como el intercambio de mensajes, es decir, un globo de alguna conversación privada (inbox), por medio de algún medio electrónico, con contenido sexual explícito 0 implícito, ya sea con texto y/o imagen creada por el autor, en donde se considere que se muestra desnudo o semidesnudo. Tomando en cuenta la teoría de Skinner como punto de partida, es necesario hacer hincapié que dentro de los mensajes pueden observarse diferentes operantes verbales, 0 sea, variables que producen algún efecto en la conducta 0 el ambiente.

La adquisición de las conversaciones para su posterior análisis se llevó a cabo mediante un perfil de Facebook, en donde los datos de identificación fueron impersonales y refiriéndose a la investigación. A partir de éste se realizó una invitación de amistad a grupos sociales de adolescentes y perfiles de éstos que indicaran su lugar de residencia en Aguascalientes.

A quienes indicaban ser menores de edad y viviendo en la ciudad se les envió un mensaje inbox con una presentación de la investigación, además de una invitación para contestar un cuestionario de tamizaje mediante el software LimeSurvey ofrecido a los alumnos de la Universidad Autónoma de Aguascalientes para realizar encuestas de diversa índole según la conveniencia del estudio a realizar, esto con la finalidad de detectar si había practica el sexting.

Dicho cuestionario obtenido de la traducción y adaptación realizada por Marrufo (2012) del cuestionario "Sex and Tech", en donde se hace referencia a la percepción general del adolescente sobre cómo se da el sexting, las acciones del sexting cometidas, su postura ante afirmaciones del fenómeno y los calificativos para actos del sexting. 
A aquellos adolescentes que indicaban haber practicado el sexting se les envió enseguida un mensaje (también vía inbox) solicitándoles su cooperación para la investigación compartiendo alguna de las conversaciones en donde se pudiera apreciar dicho fenómeno, sin embargo, al tratarse de adolescentes se consideró prudente nombrar a estas conversaciones como "de ligue":

Por otro lado, se tuvo un acercamiento personal con algunos adolescentes de la ciudad asistiendo a una institución en donde éstos acudieran a realizar diversas actividades (Casa del Adolescente) y se solicitó autorización para aplicar el cuestionario de tamizaje Tec-Sex. Después de aplicarlo, quienes indicaban haber participado en alguna conversación de tipo sexting se les solicitó su Facebook para iniciar una conversación inbox con ellos informándoles que una página de la investigación en donde había información sobre el tema los agregaría como amigos.

Las conversaciones que fueron enviadas se transcribieron con el fin de poder ser codificadas y analizadas de acuerdo al Inventario de Conductas propuesto y elaborado ex profeso ad hoc a la conducta, esto tomando como Participante A aquel que enviaba la conversación y como Participante B el interlocutor.

Para realizar el Inventario de Conductas fue necesario capacitar a un analista externo y de este modo tener dos observadores que pudieran validar el mismo. Cabe resaltar que las conductas fueron clasificadas de acuerdo a la teoría propuesta por Skinner (1981), haciendo una diferencia entre la modalidad del mensaje pudiendo presentarse como Texto, Imagen o mensaje Mixto, la forma del texto o la imagen, pudiendo ser Implícita o Explícitamente y, finalmente el tipo de transmisor de la operante verbal, es decir tomando en cuenta los mandos y reforzadores emitidos.

Con esta clasificación del tipo de mensaje se decidió implementar un código en donde se pudiera observar primeramente la modalidad, seguido de la forma y finalmente la operante verbal del interlocutor, por ejemplo, un Texto Explícito donde se realiza una Advertencia: TExAd.

La validación del Inventario se realizó con el registro de 6 conversaciones ajenas a las ofrecidas por los adolescentes. Para llegar a los acuerdos necesarios entre los observadores se construyó una matriz de confusión, misma que permitió encontrar errores en la definición de las categorías. Una vez teniendo la versión definitiva del Catálogo se realizó un registro de 7 conversaciones obteniendo un índice Kappa de .86.

Finalmente, el trabajo de investigación se realizó con 9 conversaciones de tres adolescentes mujeres con un promedio de 3 conversaciones por cada una, todas con diferentes interlocutores, mismas que fueron enviadas voluntariamente, previo consentimiento informado.

De las 9 conversaciones se realizó una codificación y análisis para establecer patrones ocultos de conducta en la práctica del sexting haciendo uso del software Theme 5 con un $p<=0.05$ además de analizarse las frecuencias de los mandos emitidos por los participantes.

Terminado el análisis se procedió a la realización de reporte de resultados encontrados, mismos que se describen a continuación.

Cabe mencionar que durante todo el proceso se tomaron en cuenta varias consideraciones éticas que fueron relevantes por tratarse de adolescentes; entre ellas se encuentran avisos de privacidad durante las conversaciones inbox, consentimiento informado entregado antes de contestar los instrumentos, leyendas de privacidad y autorización en ellos y la aclaración de que sus nombres de usuarios serían eliminados, tanto de quienes enviaron sus conversaciones como de aquellos con quienes entablaron el episodio de sexting.

\section{RESULTADOS}

Se realizaron 66 cuestionarios Tec-Sex, sin embargo se excluyeron aquellos que fueron respondidos por mayores de 18 años, teniendo un total de 39 cuestionarios válidos (21 mujeres y 18 hombres) respondidos por adolescentes de entre 12 a 17 años de edad. 


\section{SEXTING PRACTICADO POR ADOLESCENTES: SU MORFOLOGÍA EN FACEBOOK}

De las solicitudes de amistad que se enviaron, 60 fueron respondidas positivamente, sin embargo solamente a 17 adolescentes cumplieron con la edad, la residencia en Aguascalientes y la práctica del sexting de acuerdo al cuestionario de tamizaje, mismos a los que se les pidió su cooperación con el envío de conversaciones.

De las 17 solicitudes, solamente 3 fueron aceptadas y éstas fueron las que enviaron sus conversaciones para el análisis de la investigación. Las 3 participantes indicaron ver imágenes de los perfiles y leer blogs de otras personas y mandar o enviar mensajes de texto, una de ellas indicó no enviar ni recibir fotos o videos en su celular y otra de ellas aceptó enviar fotos en línea y subir videos a You Tube.

A partir de la observación de las 9 conversaciones que las tres participantes enviaron, se encontró que el promedio de mandos emitidos en un episodio de sexting fue de 15.

Además, se encontró que el mando más usado fue la Solicitud Explícita en Texto ( $x=5.5)$, seguido por la Respuestas Amplia Explícita en Texto $(x=4.25)$ y la Respuesta Simple Explícita en Texto ( $x=3.25)$; siendo los de menor frecuencia de uso la Advertencia Explícita en Mensaje Mixto, Alabanza Explícita en Mensaje Mixto, Escape Explícito en Mensaje Mixto, Respuesta Simple Sugerente en Mensaje Mixto, Escape Explícito en Texto, Gratitud Explícita en Texto, Oferta Explícita en Texto, Ruego Explícito en Texto y Mando Mágico en Texto con tan sólo una mención dentro del total de conversaciones $(x=.25)$. Es decir, hubo en promedio 5 ocasiones en que uno de los interlocutores le hizo alguna pregunta al otro para profundizar la conversación o le pidió que enviara o hiciera alguna actividad específica, pero no increpó en demasía ya que no hizo uso de la conducta rogar.

Al realizar una comparación de los mandos usados por el tipo de sujeto, es decir, las participantes que enviaron sus conversaciones y sus interlocutores, se encontró que éstos últimos tuvieron una mayor participación, totalizando 68 intervenciones con 21 mandos diferentes a diferencia de 53 actuaciones con 17 mandos diferentes de las primeras.

Por otro lado, haciendo una comparación entre la cantidad de mandos emitidos por hablante y oyente, se encontró que, de la muestra, los oyentes fueron quienes emitieron una mayor cantidad de mensajes durante el episodio de sexting.

Se realizó un análisis de secuencias de evento intra e inter sujeto, esto para conocer cómo es que se siguieron los mandos dentro de cada uno de los episodios de las participantes de acuerdo a su ocurrencia.

El análisis se realizó con una base de datos de las 9 conversaciones y se codificó con dos dígitos cada una de las intervenciones, el primero de ellos para indicar el número de participante y el segundo el número de episodio proporcionado por la adolescente.

En la tabla 2 se muestra la simbología que se utilizó para la representación gráfica de los episodios de sexting. 
Tabla 2. Simbología usada en la representación gráfica de las probabilidades de transición de episodios de sexting

Símbolo
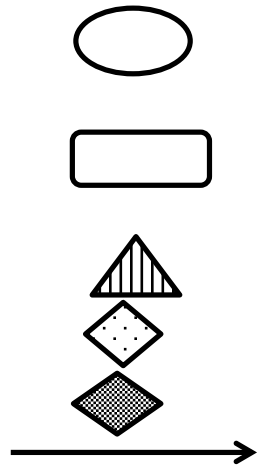

\section{Significado}

Mando emitido por el sujeto 1, es decir, la adolescente que envió la conversación.

Mando emitido por el sujeto 2, es decir, con quien mantuvo la conversación la adolescente que la envió.

Silencio

Mando emitido por el hablante.

Reforzador emitido por el oyente.

Transición simple entre mandos y reforzadores.

Transición doble entre mandos y reforzadores.

En los episodios de la primera participante fueron contabilizadas 72 transiciones entre 28 códigos presentes, de los que destacan en los que se le solicitó a la participante algún tipo de información o que realizara alguna acción (TExSo) y silencios en los que se dio por terminada la conversación y se siguió después de un día o de varias horas.

Otro dato relevante sobre dicha participante es que es ella quien tiene una mayor intervención dentro de las conversaciones y no sus interlocutores (ver figura 1).

Figura 1. Probabilidades de Transición de 4 episodios de sexting de la participante 1.
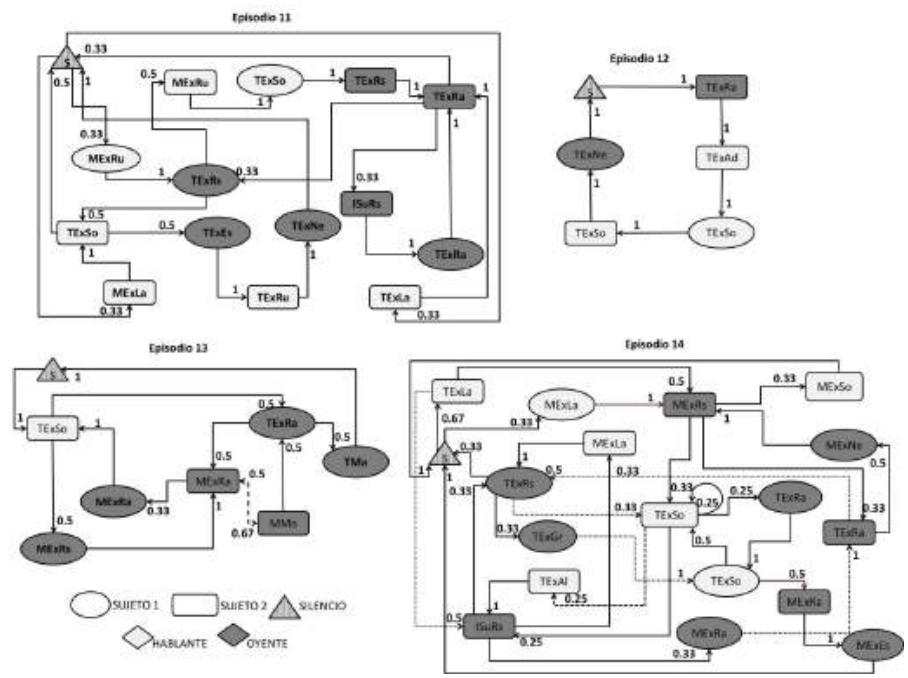


\section{SEXTING PRACTICADO POR ADOLESCENTES: SU MORFOLOGÍA EN FACEBOOK}

La segunda adolescente sólo proporcionó una conversación de un episodio de sexting que consistió en 20 transiciones de 13 códigos diferentes. Los más usados fueron los monosílabo que respondiera alguna cuestión o petición de su interlocutor (TExRS), o también aquellas respuestas que con una imagen se diera a entender, generalmente algún emoticón (TSuRs) y las Solicitudes Explícitas en Texto, es decir, peticiones o solicitudes que le increpó al otro de manera explícita (ver figura 2).

Figura 2. Probabilidades de Transición de 1 episodio de sexting de la participante 2 .

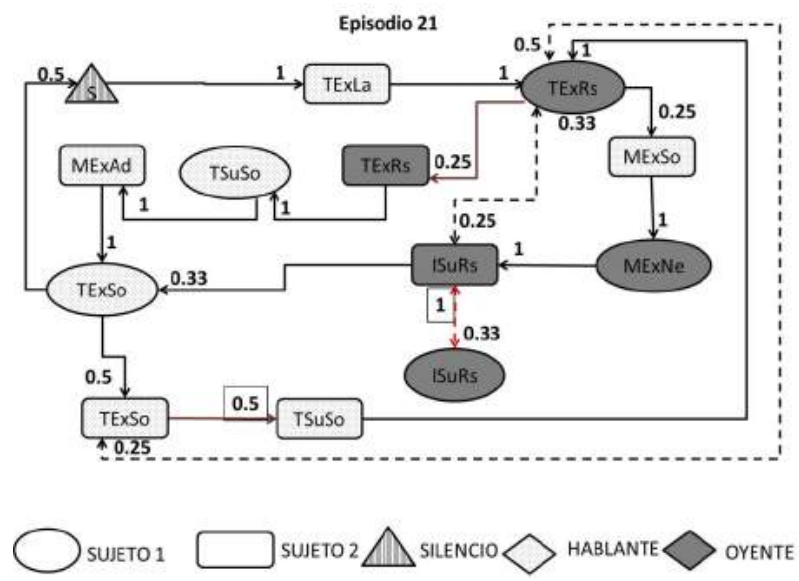

La tercera adolescente también envió cuatro episodios de sexting, sin embargo éstos sólo fueron compuestos por 42transiciones de 17 códigos diferentes. Entre los más usados fueron las respuestas en las que se hacía algún tipo de explicación o aclaración explícita a manera de texto (TExRa), las solicitudes de alguna información 0 actividad para el interlocutor de manera explícita (TExSo) y los momentos de silencio (S) en que alguno de los dos participantes no respondían (ver figura 3).

Enseguida se realizó una comparación de transiciones entre las conversaciones de la participante 1 y la participante 3, tomando en cuenta que cada una de ellas envió la misma cantidad de episodios (ver tabla 3 ).

La comparación se realizó usando una las puntuaciones z de cada una de ellas y se observó que

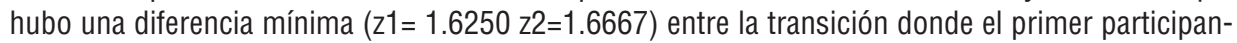
te realiza una solicitud o petición a su interlocutor y enseguida éste responde con alguna explicación o aclaración amplia (S1-TExSo - S2-MExRa), mientras que hubo una gran diferencia en la emisión de una respuesta con algún emoticón de parte de uno de ellos seguido de una respuesta con alguna aclaración de parte del otro con algún mensaje que incluyera tanto texto como imagen (S2ISuRs - S1-MExRa). 
Figura 3. Probabilidades de Transición de 4 episodio de sexting de la participante 3.

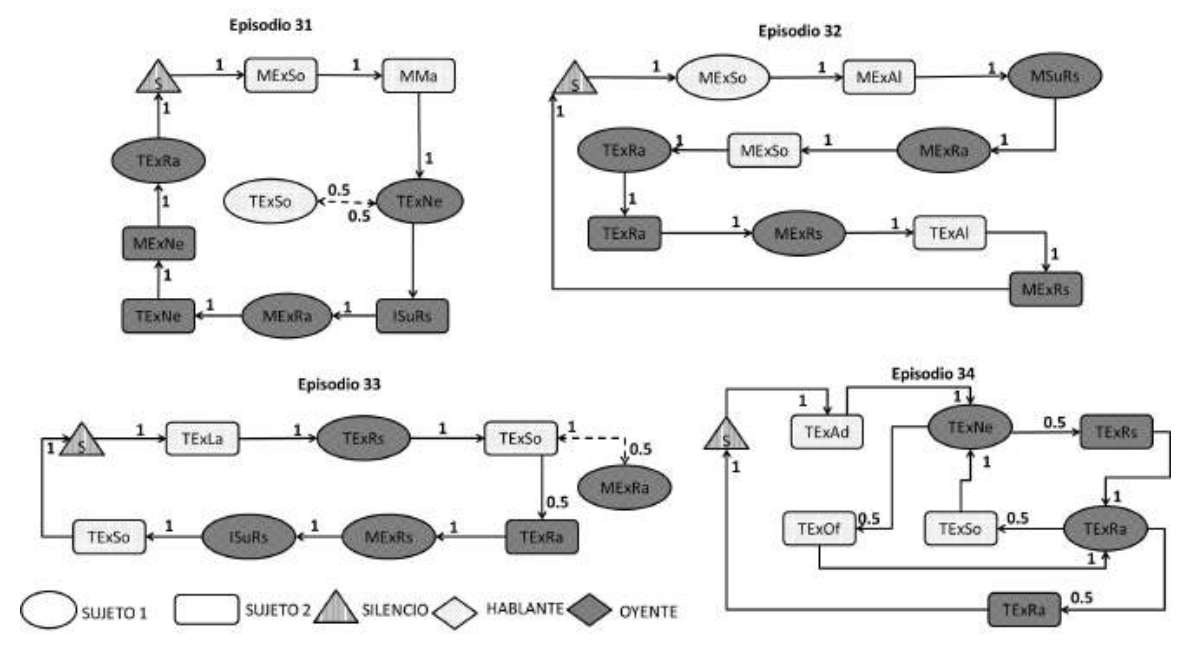

Tabla 3. Puntuaciones $z$ de probabilidades de transición de episodios de sexting de la participante 1 y la participante 3.

Transición

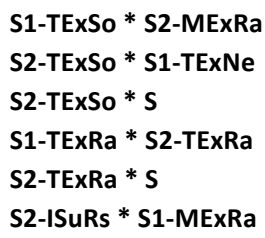

Puntuaciones z

Part. 1

1.6250

0.2722

0.2722

1.6250

0.6455

1.6250
Part. 3

1.6667

2.9200

2.9200

2.5725

1.6667

6.3246

Habiendo realizado dichos análisis se llevó a cabo la búsqueda de patrones de interacción desplegados por los participantes en un episodio de sexting, haciendo uso del software Theme 5 .

Como resultado se obtuvo que quienes enviaron sus conversaciones (Sujeto 1) no fueron quienes iniciaron sus conversaciones $(\mathrm{s} 1, \mathrm{~s})$, sino que fue su interlocutor (Sujeto 2) quienes hicieron la primera llamada de atención con algún texto (s2,texla), siendo éste generalmente un saludo (ver figura 4). 


\section{SEXTING PRACTICADO POR ADOLESCENTES: SU MORFOLOGÍA EN FACEBOOK}

Figura 4. Diagrama de patrones ocultos encontrados en conversaciones de sexting por sujeto

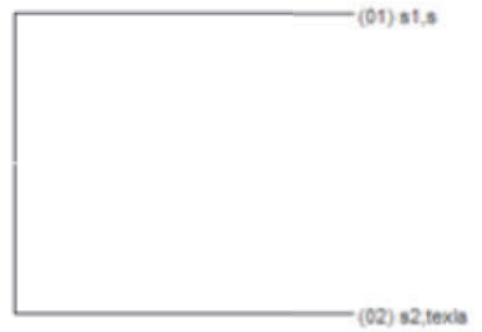

Por otro lado, tomando en cuenta la función de los participantes dentro de la conversación, es decir, tomándolos como hablante y oyente, se encontraron 2 patrones conductuales, los cuales muestran que el hablante emite una llamada de atención, generalmente un saludo, en forma de texto (ha,texla) y enseguida se emite una misma llamada de atención pero con algún mensaje que también incluya alguna imagen (ha,mexla) (ver figura 5).

El otro patrón encontrado indica que un hablante inicia la conversación con una llamada de atención explícita en forma de texto (ha,texla), después el oyente responde con un texto explícito (oy,texrs) y finalmente el hablante realiza una solicitud o petición explícita en forma de texto (ha,texso).

Figura 5. Diagramas de patrones ocultos encontrados en conversaciones de sexting por rol del participante
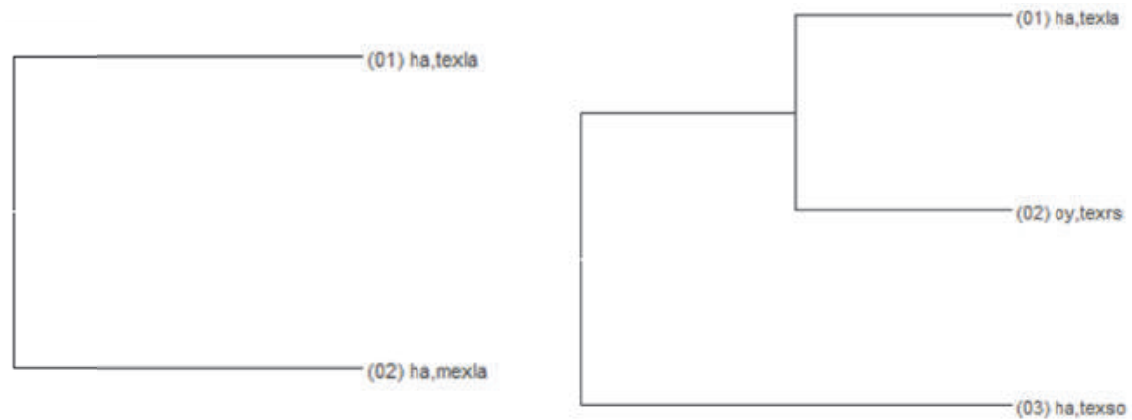

Al tratarse de un fenómeno en el que se observa el contenido sexual, tanto explícito como implícito, se tomó en cuenta que los participantes incluyen ese contenido en formas de preguntas, órdenes o sugerencias, es decir, le piden a su interlocutor intercambio de fotografías, citas o el inicio de relaciones más formales, además de información sobre sus actividades, disponibilidad y estado anímico.

En respuesta a dichas solicitudes, el interlocutor responde con alguna expresión que le permita escapar de la situación o con alguna negación explícita que no le permita tener duda a quien solicita que no obtendrá lo solicitado, además de observarse también respuestas que ponen de manifiesto interés y coqueteo e incluso hubo quien desvió la atención de manera explicativa. 
Por dichas observaciones se puede considerar a la Solicitud como el mando principal dentro de una conversación de sexting, ya que es justamente este mando el que pone de manifiesto el interés que tiene el interlocutor de intimar con el otro.

\section{DISCUSIÓN}

Habiendo realizado el análisis completo y observado que los mandos más usados en los episodios de sexting observados fueron la Solicitud, tanto de información personal o fotografías; la Respuesta Amplia como explicaciones y aclaraciones sobre la situación discutida; la Respuesta Simple generalmente con algún monosílabo o algún emoticón que indicara el estado de ánimo del participante, y la Negación explícita a realizar alguna actividad u ofrecer alguna información; se puede concluir que las conversaciones de sexting que se realizan entre los adolescentes son generalmente sin intenciones de agraviar a su interlocutor; además de ser conversaciones en las que se establecen códigos entre los participantes y que permiten un desarrollo de habilidades de comunicación por medios electrónicos.

Con ello se puede llegar a una descripción del sexting como una conducta de interacción verbal textual dinámica, por medio de TICs, en donde se encuentra involucrado un código moldeado por la comunidad de usuarios, además de un lenguaje no verbal observado en el uso de emoticones, donde también se pone de manifiesto la creatividad del usuario para generar cambios de contexto acordes a los reforzadores ofrecidos por el oyente. Todo esto llevado a cabo en una relación de intercambio coordinado (compartir).

La apertura y facilidad que ofrecen las redes sociales para que los usuarios guarden sus conversaciones, sin importar el momento en el que se realizan, permite a los adolescentes desarrollar una habilidad para tener conversaciones en tiempo diferido y comportarse de tal manera que tienen la confianza de interrumpir su comunicación sin necesariamente obtener una represalia por no enviar una respuesta inmediata.

Algo que llamó la atención en el momento de realizar la investigación, específicamente cuando se hizo el filtro a los usuarios de Facebook, es la veracidad de los datos que se publican en la red social, especialmente lo indicado por los adolescentes en los apartados "Información general" y "Formación y empleo". La información que más se manipula es la referida a la fecha de nacimiento, los lugares de residencia, los lugares de escuelas y empleos pasados. Este manejo de información y sobre todo la falsedad de ella en cuanto a la auto presentación de los usuarios, ya mencionada por Di Próspero (2011) y por Krämer y Winer (2008) genera la posibilidad de entablar relaciones diferentes a las que podrían entablar con sus datos reales como lo indica (Farber et al., 2012), conduciendo a un factor de riesgo contextual.

En cuanto a la privacidad de la información y de las mismas conversaciones se puede observar la apertura de visibilidad que algunos usuarios tienen en sus perfiles, solamente unos cuantos tienen un dominio de la normatividad y las herramientas que la red social ofrece.

Uno de los factores de riesgo visibles en las interacciones que se tuvieron con los adolescentes durante todo el proceso fue la importancia que le dan los adolescentes a las aprobaciones de sus pares por medio de comentarios favorables o likes tanto de las publicaciones como de las fotografías, post y links que "suben" a la Internet, generando que, sobre todo fotografías tituladas como "sexis" se vuelvan pupularmente públicas.

Teniendo en cuenta todas estas características de la práctica del sexting, se considera las variantes en la comunicación e interacción que actualmente tienen los adolescentes, además de la apertura que éstos tienen hacia las TICs deben ser consideradas y puestas a discusión en la generación de Políticas Públicas. 


\section{SEXTING PRACTICADO POR ADOLESCENTES: SU MORFOLOGÍA EN FACEBOOK}

Otra de las características que se resalta en este estudio es la participación que tienen tanto hombres como mujeres en la práctica del sexting, resultado que debiera también considerarse en la formulación de leyes que protejan a ambos sexos, considerando que sin importar éste los adolescentes se enfrentan a los mismos factores de riesgo en nuestra sociedad cada vez con mayor apertura e inclusión.

Finalmente se puede decir que el mayor impacto que se puede tener en los adolescentes es en la prevención de todas las consecuencias de éste fenómeno, considerando sobre todo el autocuidado, mismo que aumenta al tener conocimiento fidedigno del proceso que se observa en su práctica y las contingencias que refuerzan o castigan dicha conducta.

Es necesario tomar en cuenta que el presente estudio tiene limitaciones importantes como la poca cantidad de conversaciones y el número de transiciones que en ellas se observan; la falta de contexto de los episodios de sexting y de información de los interlocutores como el tipo de relación entre ellos y los diferentes códigos que entre ellos existen; falta de información del proceso de seducción que describe Mantovani (2001) entre los participantes y la falta de medición de tiempo y latencia en las respuestas de las conversaciones para hacer una mejor descripción de las habilidades de los participantes.

Sin embargo, los resultados se presentan como un inicio en la investigación sobre conversaciones de tipo sexual en las redes sociales y, con esto, se pretende tener un mejor conocimiento del tipo de participación que en ellas se tiene, esperando ser una aportación para la mejor construcción y delimitación de dicho fenómeno que no tiene hasta el momento un consenso local, nacional 0 internacional para su definición.

\section{REFERENCIAS}

Anderson B., Fagan P., Woodnutt T. y Chamorrro-Premuzic T. (2012) Facebook psychology: popular questions answered by research. Psychology of Popular Media Culture. Vol. 1, Num. 1 23-37 DOI: 10.1037/a0026452

Anguera M. T. y Hernández-Mendo A. (2014) Técnicas de análisis en estudios observacionales en ciencias del deporte. Cuadernos de Psicología del Deporte. Vol. 15, Num. $113-30$

Arab L. E. y Díaz G. A. (2015) Impacto de las redes sociales e internet en la adolescencia: aspectos positivos y negativos. Revista Medica Clinica Las Condes. Vol. 26, Num. 1 07-13

Ardila R. (2007) Verbal behavior de B.F. Skinner: su importancia en el estudio del comportamiento. Revista Brasileira de Terapia Comportamental e Cognitiva. Vol. 9, Num. 2, 195-197

Delevi R. y Weisskirch R. S. (2013) Personality factors as predictors of sexting. Computers in Human Behavior. Vol. 29 2589-2594 Recuperado de: http://dx.doi.org/10.1016/j.chb. 2013.06.003

Drouin, M., Vogel, K. N., Surbey, A. y Stills, J. R. (2013) Let s talk about sexting, baby: computer mediated sexual behaviors among young adults. Computers in Human Behvior, 29, doi.org/10.1016/j.chb.2012.12.030

Farber, B. A., Shafron G., Hamadani J., Wald E., y Nitzburg G., (2012) Children, Technology, Problems, and Preferences. Wiley Periodicals, Inc., Journal of Clinical Psychology: In Session, 68 (11), 1225-1229.

Lounsbury, K., Mitchell, K. J., Finkelhor, D., (2011) The true prevalence of Sexting. Children Crimes Against Research Center, University of Hampshire, Abril 2011.

Marrufo, M. R. 0. (2012) Surgimiento y proliferación del Sexting. Probables causas y consecuencias en adolescentes de secundaria. Trabajo de investigación realizado para obtener el grado de Maestro en Investigación Educativa. Universidad Autónoma de Yucatán. México 
Peña-Correal T. y Robayo-Castro B. H. (2007) Conducta verbal de B. F. Kinner: 1957-2007. Revista Latinoamericana de Psicología. Vol. 39, Num. 3 653-661 Recuperado de: http://www.redalyc.org/articulo.oa?id=80539314

Santoyo, V. C. y Espinosa A. M. C. (1991) Decisiones metodológicas para el análisis contextual de la Interacción Social. Revista Mexicana de Análisis de la Conducta, 17 (3) 85-103.

Skinner, B. F. (1979) Contingencias de reforzamiento. Un análisis teórico. Tr. Edgar Galindo Cota. Editorial Trillas. México.

Skinner, B. F. (1981) Conducta verbal. Trillas. México

Wolak, J. y Finkelhor D., (2011), Sexting: A typology. Crimes Against Children Reserch Center. University of New Hampshire, Bulletin March 2001, Recuperado de http://www.unh.edu/ccrc/pdf/CV231_Sexting\%20Typology\%20Bulletin_4-6-11_revised.pdf

Yang, L. (2015) Adolescent sexual behavior in the digital age: considerations for clinicians, legal professionals and educators. Journal Youth Adolescence, Online, DOI 10.1007/s10964-015-02851

Zemmels, D. R. y Khey, D. N. (2014). Sharing of digital visual media: privacy concerns and trust among young people. Am J Crim Just. DOI 10.1007/s12103-014-9245-7 
